Supporting Information

\title{
Ionic liquid passivation eliminates low- $n$ quantum well domains in blue quasi-2D perovskite films
}

\author{
Ningning Zhu ${ }^{1,2}$, Kaixuan $X u^{3,4}$, Jun Xing ${ }^{1 *}$, Jun Zhang ${ }^{3,4,5}$, Jiangnan Dai ${ }^{*}$
}

${ }^{1}$ Key Laboratory of Eco-Chemical Engineering, Ministry of Education, College of Chemistry and Molecular Engineering, Qingdao University of Science \& Technology, Qingdao 266042,

China.

${ }^{2}$ Wuhan National Laboratory for Optoelectronics, Huazhong University of Science and Technology, Wuhan 430074, China.

${ }^{3}$ State Key Laboratory of Superlattices and Microstructures, Institute of Semiconductors, Chinese Academy of Sciences, Beijing,100083, China

${ }^{4}$ Center of Materials Science and Optoelectronics Engineering, University of Chinese Academy of Sciences, Beijing 100049, China

${ }^{5}$ Beijing Academy of Quantum Information Science, Beijing 100193, China

\section{"Corresponding Author}

xingjun@qust.edu.cn (J.X.)

daijiangnan@hust.edu.cn (J. D.) 
a
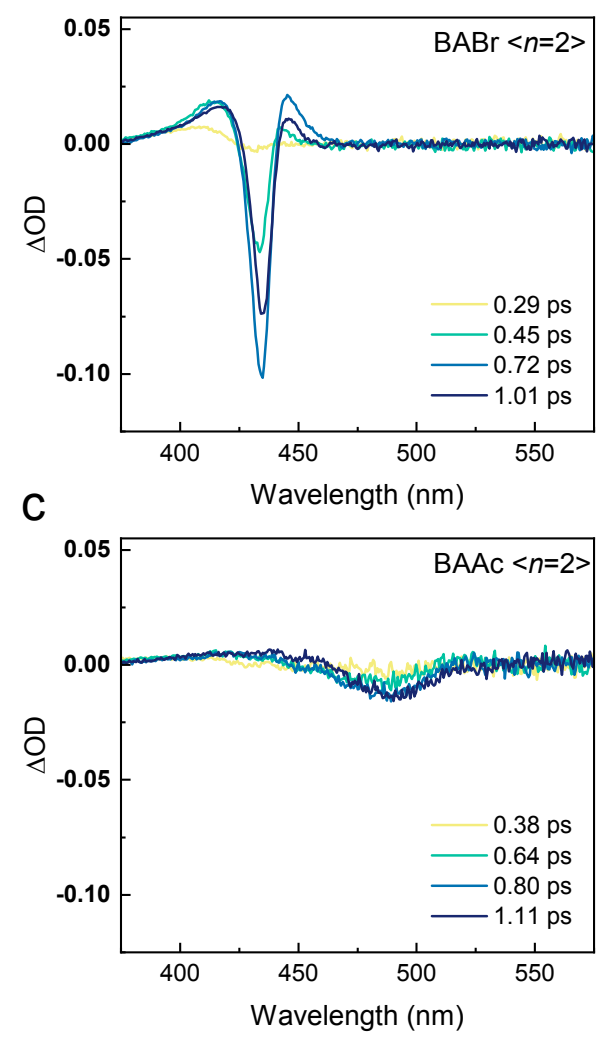

b
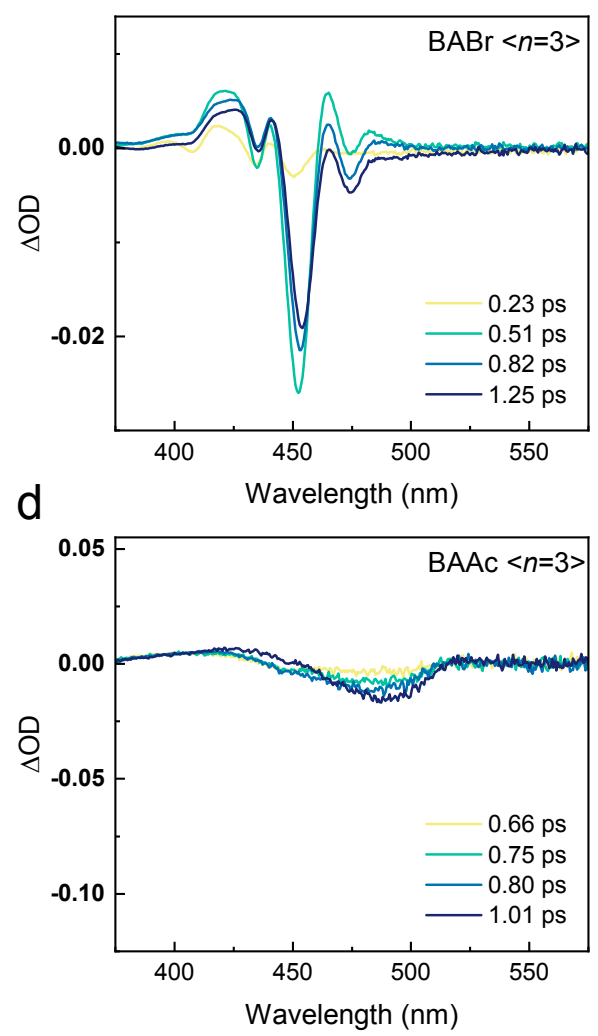

Figure S1 TA spectra of the (a) $<n=2>\mathrm{BABr}$-derived, (b) $<n=3>\mathrm{BABr}$-derived, (c) $<n=2>$

BAAc-derived, (d) $<n=3>$ BAAc-derived perovskite films. 


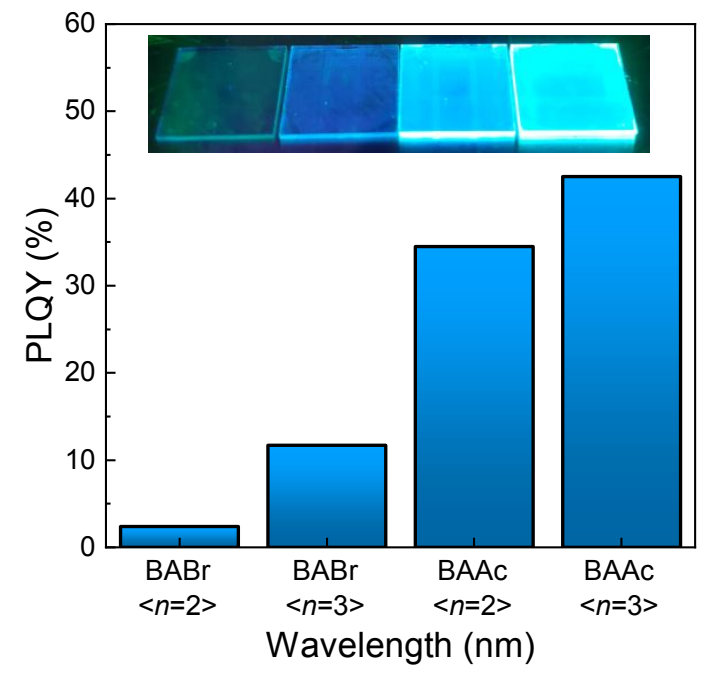

Figure S2 The PLQY of $<n=2>$ and $<n=3>$ BABr-derived and BAAc-derived perovskite films. 
a

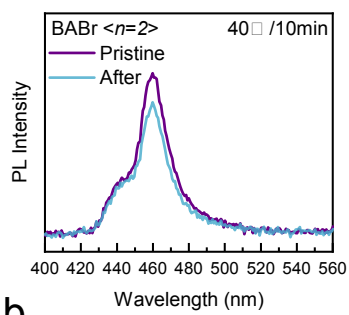

b

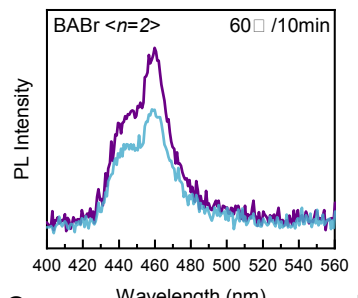

C

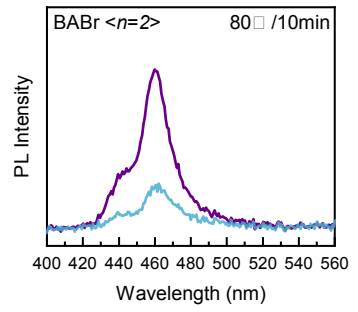

d
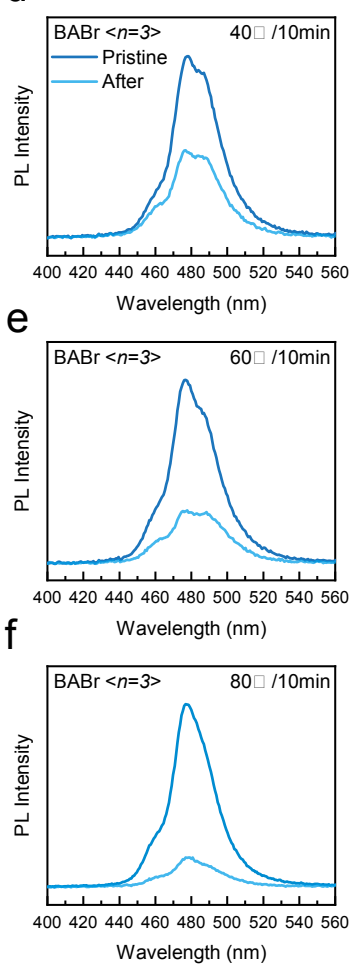

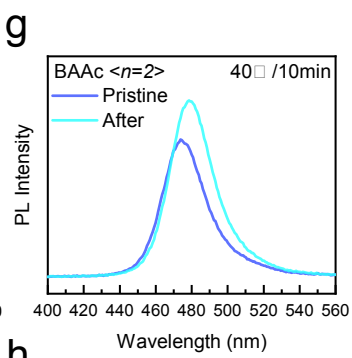

$\mathrm{h}$
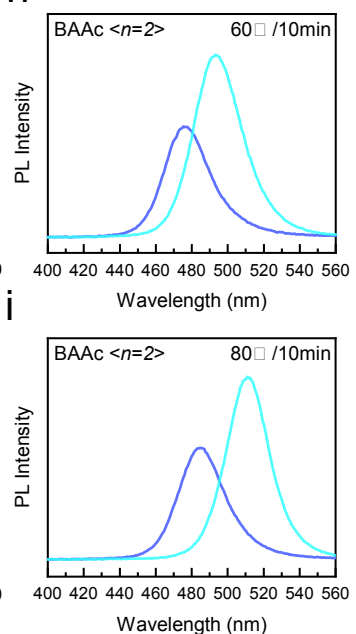

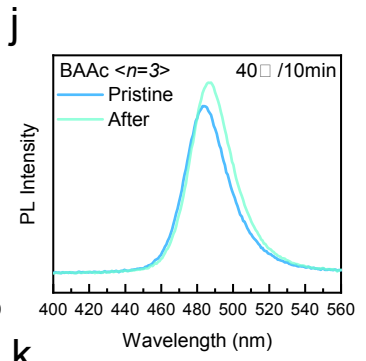

$\mathrm{k}$
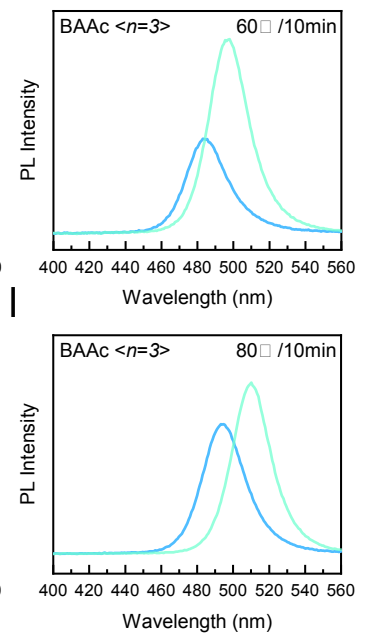

Figure S3 PL spectra of (a-c) $<n=2>$ BABr-derived, (d-f) $<n=3>$ BABr-derived, (g-i) $<n=2>$

BAAc-derived, (j-l) $<n=3>$ BAAc-derived perovskite films annealing at $40,60,80^{\circ} \mathrm{C}$ for 10 minutes. 
a

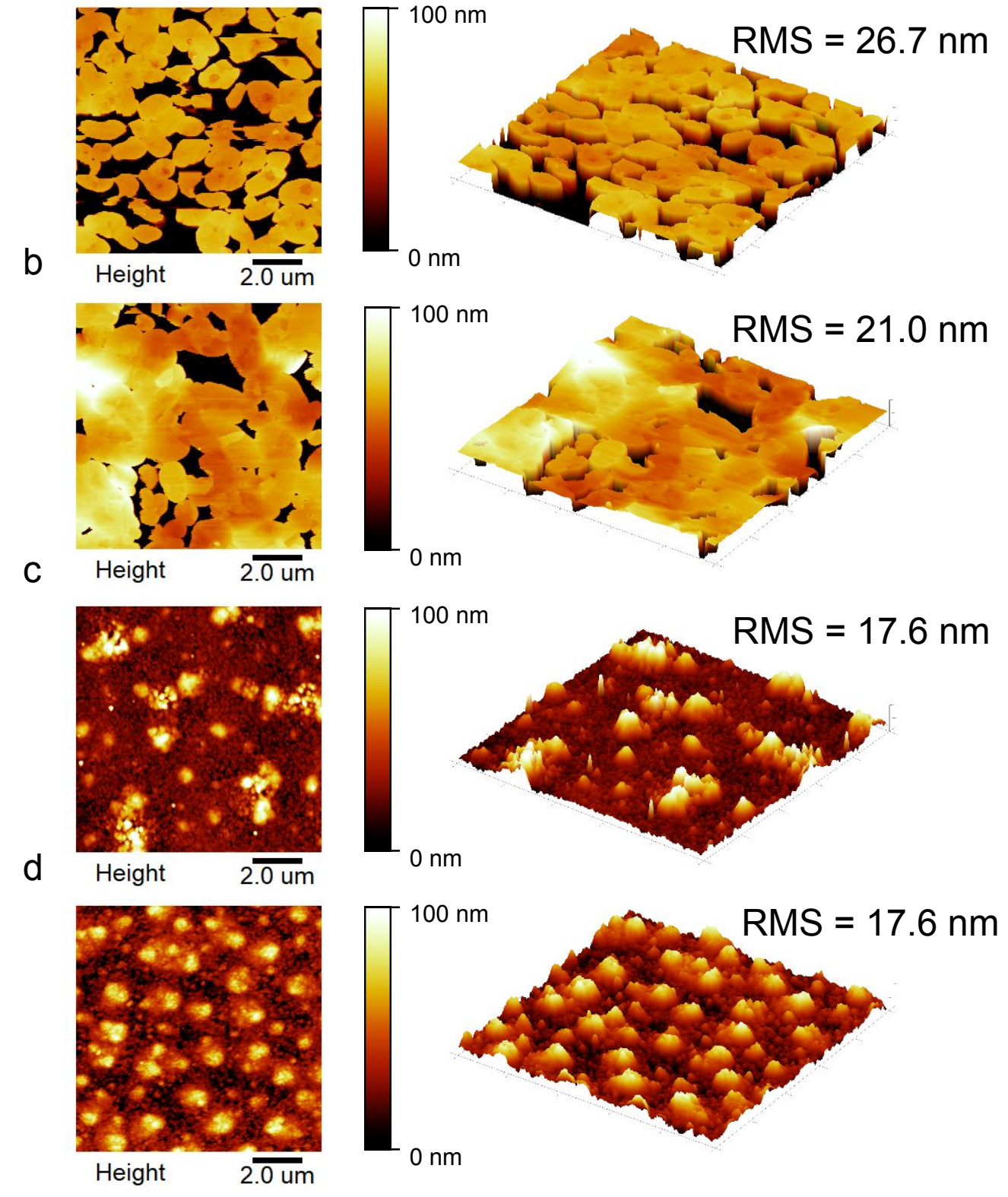

Figure S4 AFM images of (a) $<n=2>$ BABr-derived, (b) $<n=3>$ BABr-derived, (c) $<n=2>$ BAAc-derived, (d) $<n=3>$ BAAc-derived perovskite films. RMS means Root-Mean-Square. 

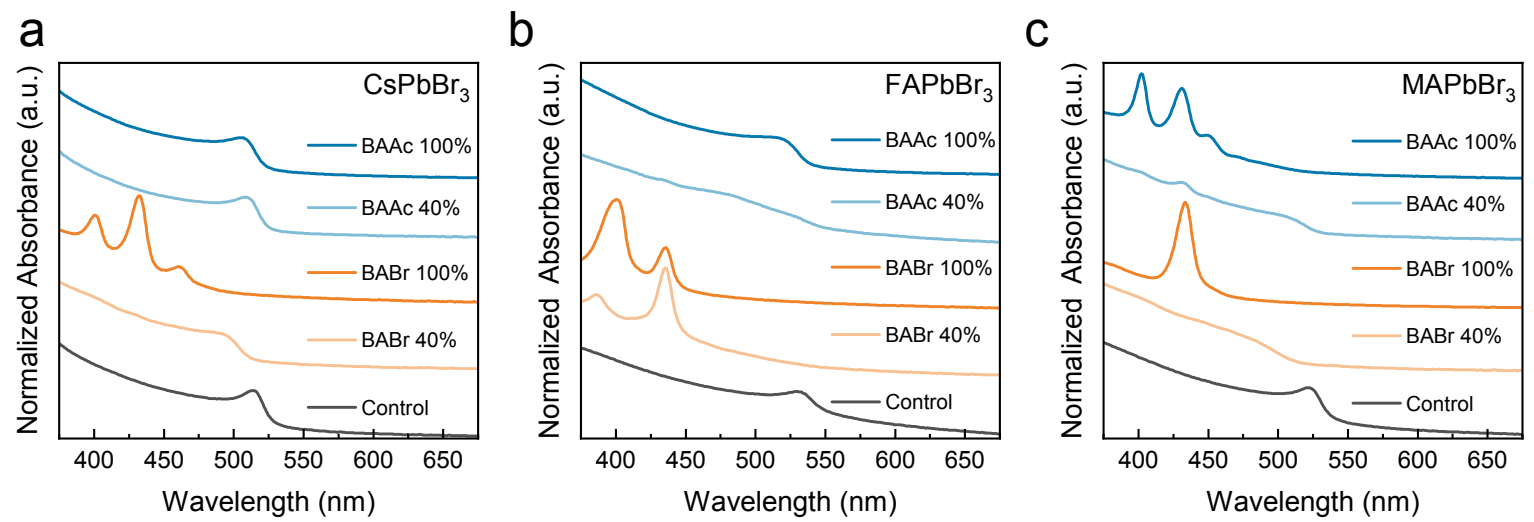

Figure S5 The steady absorption spectra of $40 \%$ and $100 \%$ ratio BABr-derived and BAAc-derived perovskite films on (a) Cs based perovskite, (b) FA based perovskite and (c) MA based perovskite. 

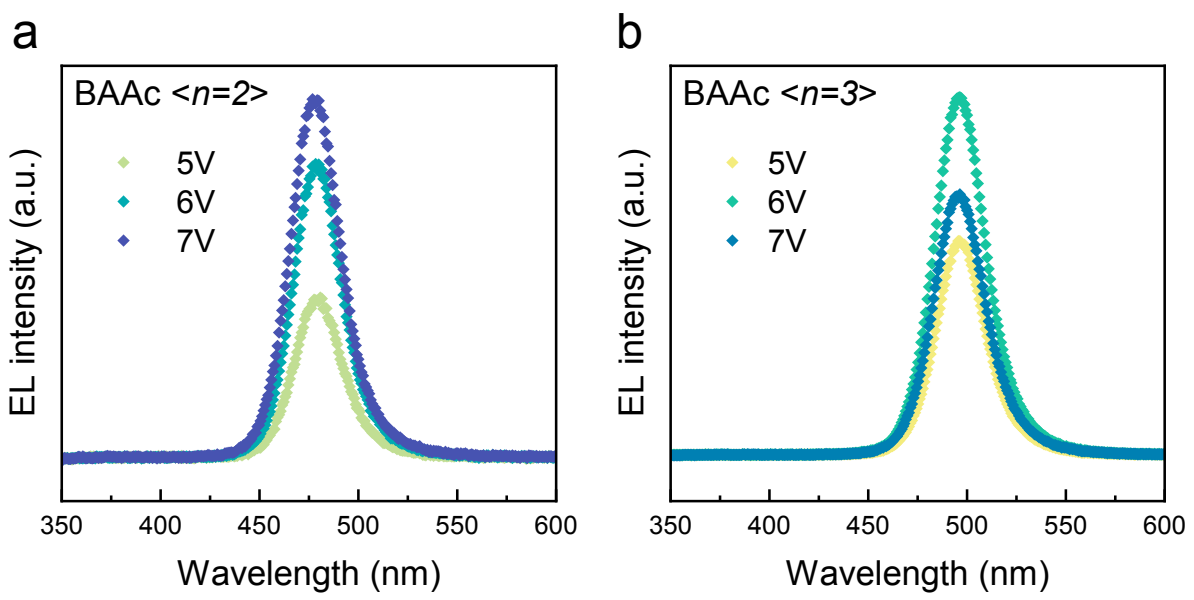

Figure S6 EL spectra of the (a) $<n=2>$ and (b) $<n=3>$ BAAc-derived PeLEDs under bias of 5, 6 and $7 \mathrm{~V}$. 


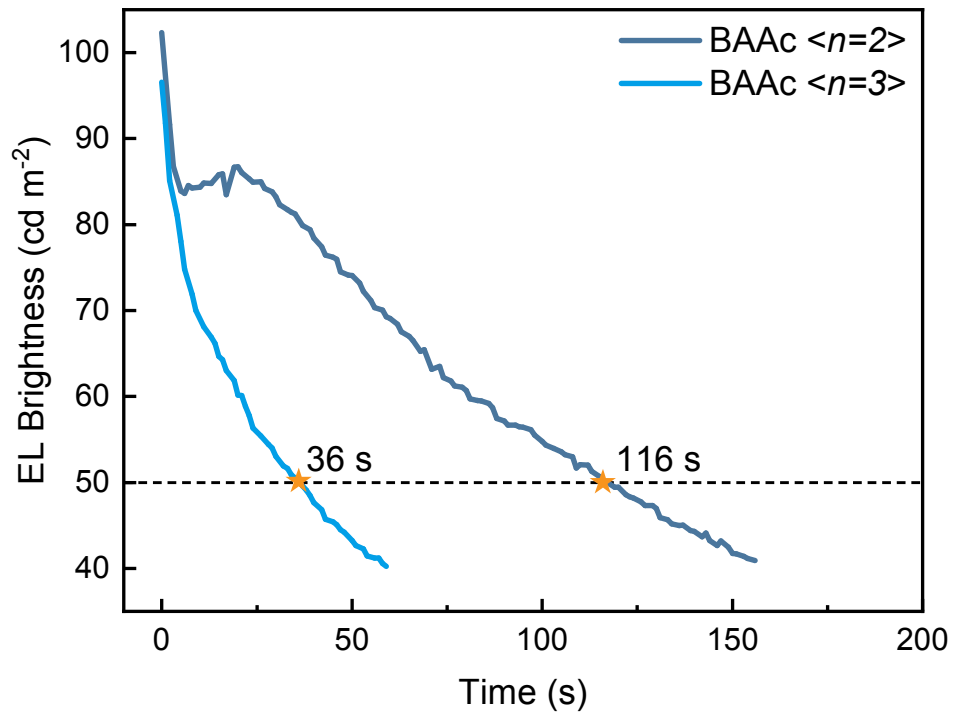

Figure S7 Operational lifetime of the $<n=2>$ and $<n=3>$ BAAc-derived PeLEDs with an initial luminance of $100 \mathrm{~cd} \mathrm{~m}^{-2}$. 